\title{
Charge reduced nanoparticles by sub-kHz ac electrohydrodynamic atomization toward drug delivery applications
}

\author{
Van Thanh Dau ${ }^{1 *}$, Tuan-Khoa Nguyen ${ }^{2)}$, and Dzung Viet Dao, ${ }^{1,2)}$ \\ ${ }^{1}$ School of Engineering and Built Environment, Griffith University, Gold Coast, 4215, Australia \\ ${ }^{2}$ Queensland micro and nano technology centre, Griffith University, Brisbane, 4111, Australia
}

\begin{abstract}
In this letter, we present an electrohydrodynamic atomization (EHDA) technology that generates and delivers charge reduced nanoparticles to open space without collector electrode. The backward ring-nozzle ac EHDA system driven at sub-kHz frequencies generates alternatively charged particles which exert electric force and recombine in the vicinity of the spraying electrode. This unique configuration creates a stable jet stream of charge reduced nanoparticles, to the contrary of classical dc EHDA systems. Experiments indicate that nanoparticles are emitted through a wide range of voltages and frequencies, match up to the hydrodynamic time of the cone jet model. These unique advantages of the new system would empower the nanoparticle EHDA devices for aerosol drug delivery in bio and health care applications.
\end{abstract}

EHDA or electrospray, with a unique capability of ejecting nanometer-scale particles, has emerged as a promising technique for applications, such as nanomedicine ${ }^{1-4}$, bioprinting ${ }^{5,6}$ and ionization mass spectroscopy ${ }^{7-9}$. When conductive liquid in a capillary is subjected to a strong electric potential, the accumulated surface charge at the outer interface leads to radial electrostatic pressure. The liquid volume at the capillary tip decreases and the liquid deforms into a conical shape called Taylor cone ${ }^{10}$. The EHDA occurs when the liquid eventually emits into a unidirectional jet through the capillary tip and breaks into a monodispersed cloud of highly charged particles ${ }^{11}$. The critical voltage for EHDA formation is proportional to the liquid surface tension $\gamma$, and there is a balance between capillary pressure and the normal electrostatic pressure at the conical liquid surface $\gamma / r=\varepsilon_{0} E^{2} / 4$, where $r$ is local capillary radius, $E$ is the electric field and $\varepsilon_{0}$ is the permittivity of vacuum.

The current interests in inhalable drug delivery are the generation and delivery of micro/nanoparticle as carriers for nanomedicine $^{12}$. A typical EHDA system consists of a spray electrode atomizing charged liquid and a reference electrode placed downstream to form an electric field and to define the movement of the generated product ${ }^{13}$. Micro/nano particles are generated with either type of dominant charge (positive or negative). In either case, the system generates spray plume toward its reference electrode and the majority of particles will land on the reference electrode, or toward the earthed surrounding due to electrostatic force. In respiratory drug delivery, charged particles of the drug made by EHDA would land on the walls of the

\footnotetext{
${ }^{*}$ Corresponding author: v.dau@griffith.edu.au
} 
respiratory system instead of reaching deep in the lung ${ }^{14}$. In order to utilize the produced particles for bio or health care applications, charge reduction to produce neutral clusters of selected compositions is imperative. This was addressed by various methods. For instance, dc EHDAs with parallel electrospray configurations of dual spray sources mixing two oppositely charged clouds of droplets from nozzles supplied with positive and negative voltage ${ }^{15-17}$, or gaseous ions generated by corona discharge neutralizing the electrospray droplet ${ }^{18}$. For ac EHDAs, works have paid attentions to high frequency (i.e. from 10 $\mathrm{kHz}-10 \mathrm{MHz}$ ), in which micron-sized particles are generated by resonating meniscus at the orifice ${ }^{19-21}$. As the electrostatic forces weaken in the space between the extractor and needle electrodes, an external air stream can convey the particles through the channel, then they are collected and prepared via several interim steps before being distributed to a target of application by another technology 22,23 .

In this letter, we present an EHDA backward ring - nozzle setup operating with a sub-kHz frequency alternating voltage. By the recombination of oppositely charged particles, a stable jet stream of nanoparticles is generated with much smaller electrical charge and moves away from both electrodes. Somewhat counterintuitively, this forward spray is feasible by the matching of the initial particle's momentum with the alternating of the reversed electric field, and the existence of virtual electrode due to the space charge effect. The experiments show that test liquid, isopropyl alcohol, could be steadily sprayed via the cone jet mode at voltage levels of $\sim 4 \mathrm{kV}(\mathrm{p}-\mathrm{p})$ and frequency range $\sim 100 \mathrm{~Hz}$, with the particles not attracting to the ring electrode. Unlike other studies on high-frequency ac EHDA (e.g. > $10 \mathrm{kHz}$ ) in conventional nozzle - collector setup, our concept uses sub-kHz frequency, generates low net charge nanoparticles and would be beneficial to pulmonary nanomedicine delivery since the mist of nanoparticles are delivered without the restriction of either the collector or the assistance of external flow.

Figures 1 shows our ac EHDA concept consisting of a concentrically placed ring and capillary. The ring is located upstream at a distance $d$ from the capillary tip and is grounded, while an ac voltage is applied to the capillary. Since the conductivity is discontinued at the surface between liquid and air, charge accumulation causes the liquid surface to protrude out of the capillary and to emit particles via the Taylor cone. The differential electric fields at the capillary tip, normal to the capillary cross-section, drive the droplets forward. At very low frequencies or in the dc mode, this field will reverse the direction of particles and force them back towards the ring electrode as shown in the FEA model, Fig. 1(b). If the electric field changes its direction periodically, the oppositely charged particles from the previous half-cycle will exert an additional electrostatic force (i.e. space charge effect) to the tip of the spray electrode. However, at low switching frequencies, the particles have sufficient time to move away from the spray electrode, and this additional effect is negligible. 

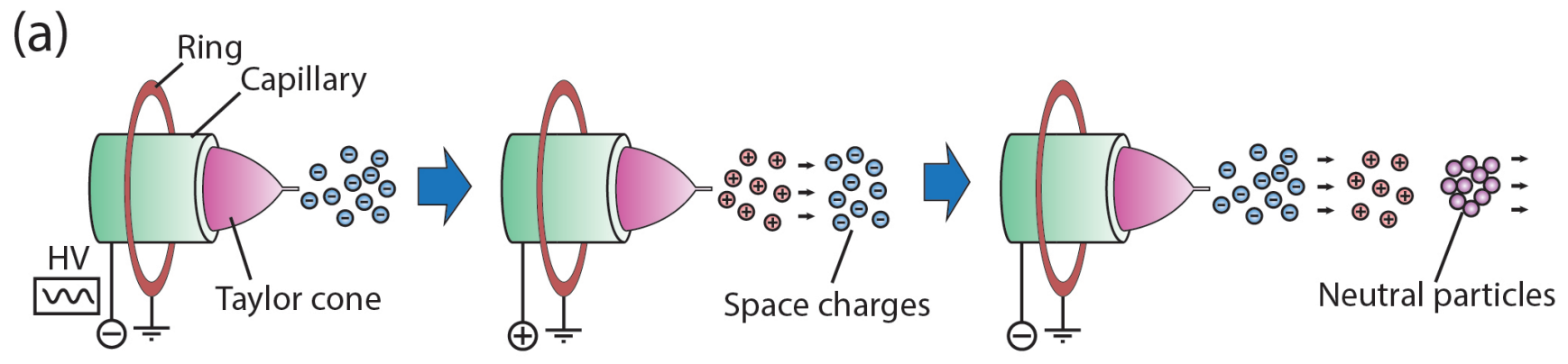

(b)

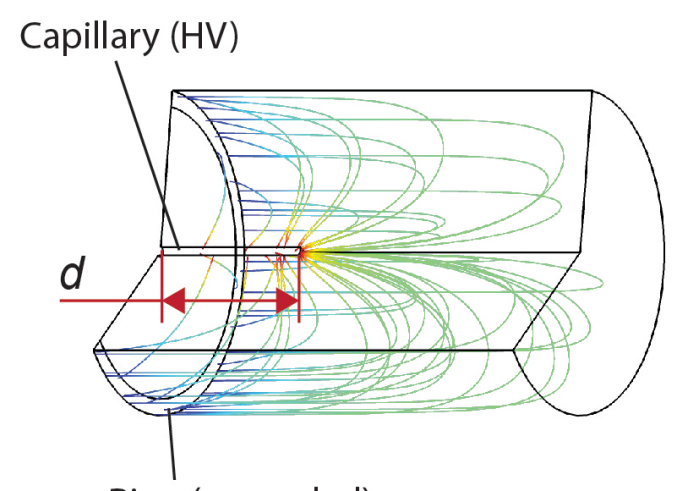

Ring (grounded) (c)

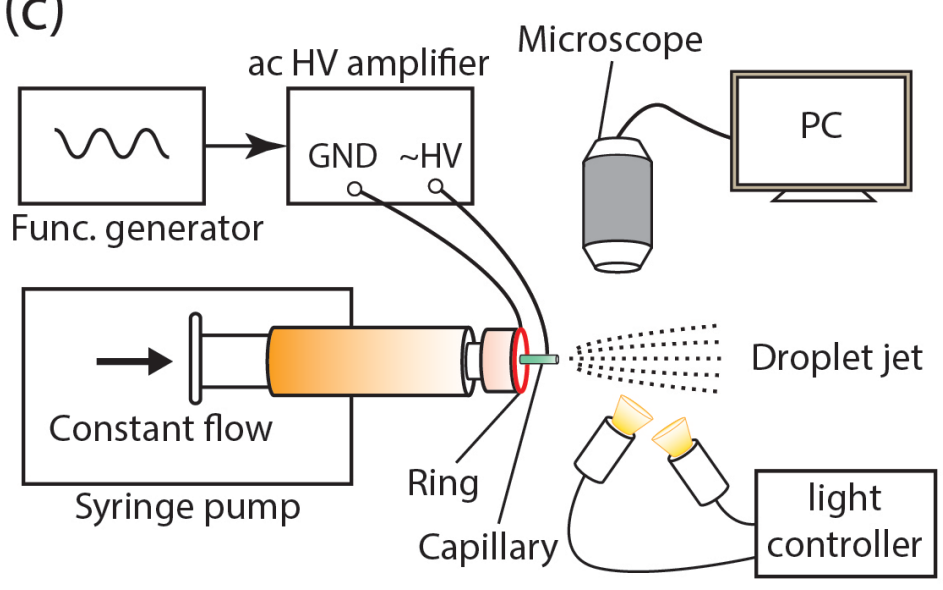

Figure 1. (a) The generation of a virtual electrode via space charge effect, the resulting charge reduced droplets form a monodispersed jet (not to scale). (b) Instantaneous electric field by FEA modelling, (c) Schematic sketch of ac driven EHDA experimental apparatus.

At suitable switching frequencies, when the atomized particles are still in the vicinity of the spray electrode tip, the accumulated space charge and the reversed electric field together play a significant role. They further decelerate the momentum of the particles from the previous half-cycle while accelerates the momentum of the particles in the following half-cycle. Therefore, the newly produced particles can catch up with their predecessors. The instantaneous mixing between positively and negatively charged particles eliminates the effect of electrostatic force, and the particles move forward based on their high momentums, Fig. 1(a).

In the experiment (Fig. 1(c)), images and video were recorded by an optical observation system using a digital microscope (Dino-lite EDGE ${ }^{\mathrm{TM}}$ ) with a maximum magnification of $200 \mathrm{X}$ and a controlled light source (LED fiber optic illuminator) generating the necessary backlight illumination. The captured images and video were displayed in real time using the microscope accompanied software. The test liquid is isopropyl alcohol (Sigma-Aldrich 99.5\%), with surface tension $\gamma \sim 20.8$ $\mathrm{mN} / \mathrm{m}$, density $\rho \sim 0.785 \mathrm{~g} / \mathrm{ml}$, viscosity $\mu \sim 1.66 \mathrm{mPas}$, conductivity $K \sim 6 \mu \mathrm{S} / \mathrm{m}$ and relative permittivity $\varepsilon \sim 18.6$. The flow rate of injection, approximated by $Q \sim \gamma \varepsilon \varepsilon_{0} / \rho K \sim 2.48 \mathrm{ml} / \mathrm{h}$ (where $\varepsilon_{0} \sim 8.85 \mathrm{pF} / \mathrm{m}$ ), was kept at $1 \mathrm{ml} / \mathrm{h}$ to generate a stable 
electrospray jet. Consequently, the largest droplet size in steady Taylor cone jet mode is estimated by scaling law $d=$ $d_{0}\left(Q / Q_{0}\right)^{1 / 2}<3 \mu \mathrm{m}$, with $d_{0}=\left[\gamma \varepsilon^{2} / \rho K^{2}\right]^{1 / 3}$. The experiment was conducted at temperature $20-22^{\circ} \mathrm{C}$, relative humidity 55 - $85 \%$ and atmospheric pressure.

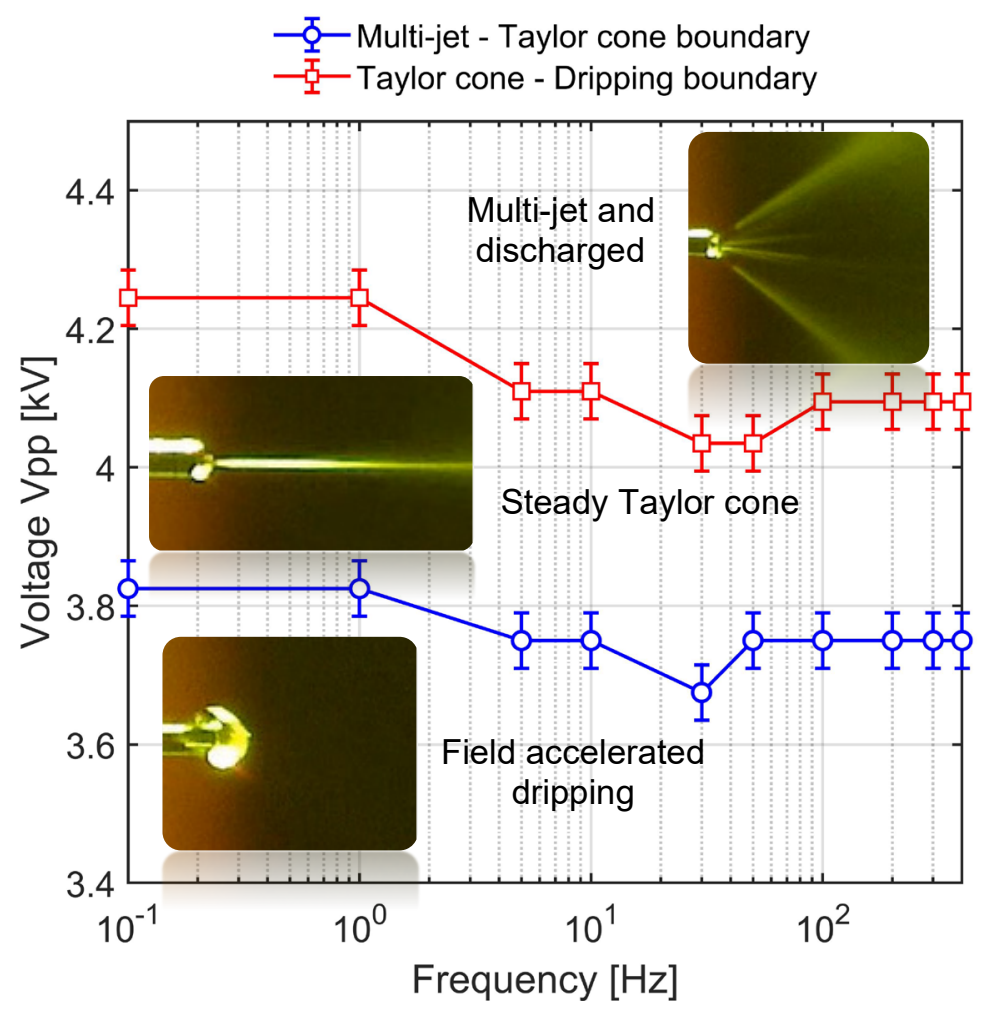

Figure 2. Voltage-frequency phase diagram with three modes for ac EHDA spraying isopropanol alcohol at the frequency range of $\mathrm{dc}(\mathrm{OHz})$ to $500 \mathrm{~Hz}$ : the upper voltage boundary is between multi-jet discharge and steady Taylor cone jet regimes, the lower voltage boundary is between the steady Taylor cone jet and field accelerated regimes. Steady Taylor cone formation and jet stream were observed at a relatively broad range of sub-kHz frequency.

Figure 2 shows the voltage-frequency diagram with three different phases including field accelerated dripping, the steady Taylor cone, and multi-jets. At a given frequency, the voltage region for the steady Taylor cone mode was confined between two boundaries consisting of the upper for the multi-jets and the lower for dripping modes, respectively. It can be seen that the formation of the steady Taylor cone was feasible at a relatively broad range of voltage and frequency, for instance, across the frequency dc $(0 \mathrm{~Hz})-450 \mathrm{~Hz}$ with a voltage from $3.8 \mathrm{kV}-4.1 \mathrm{kV}$. 

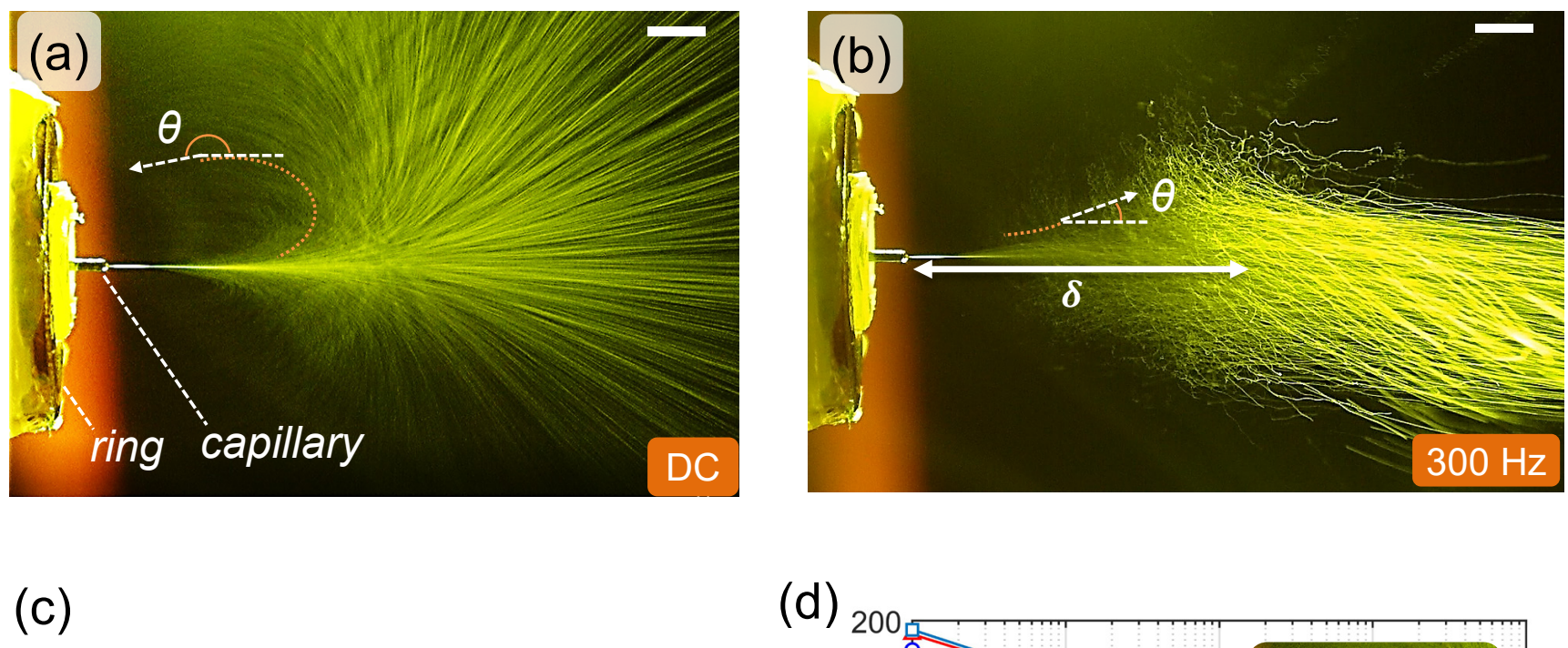

(d)
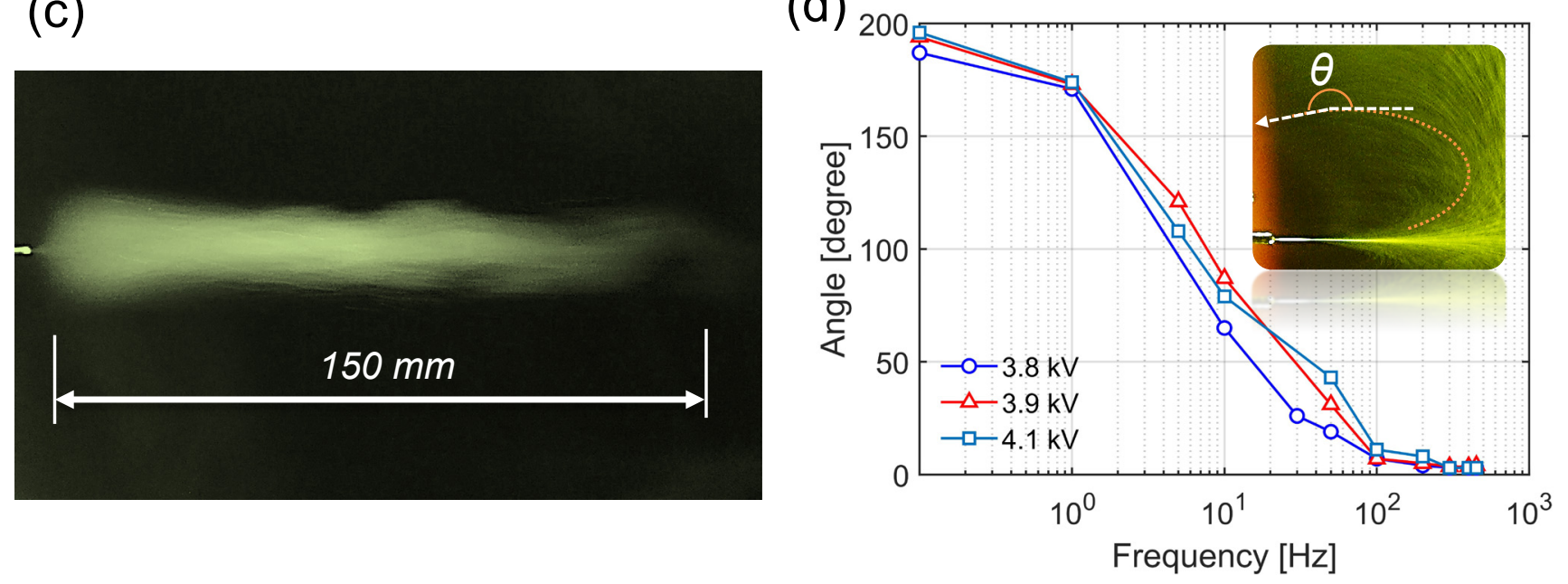

Figure 3. Optical images of the motion of the particles with (a) dc $4.2 \mathrm{kV}$ : particles fly back to the grounded ring, (b) ac $300 \mathrm{~Hz}, 4.2 \mathrm{kV}$ : particles move away from capillary (scale bar $2 \mathrm{~mm}$ ), (c) jet stream of sprayed particles under ac mode (zoomed out from (b), the capillary tip is seen at the left), and (d) angle of the jet stream $\theta$ versus frequency ranging from dc to $450 \mathrm{~Hz}$, at voltage Vpp of $3.8,3.9$, and $4.1 \mathrm{kV}$.

Figure 3 depicts the dependence of the direction of the sprayed jet stream versus the driving frequency. For quantification, we defined the motion angle $\theta$ from the trajectory of outermost particles visible under the experimental condition (Fig. 3(a)). At low frequency range below $10 \mathrm{~Hz}$, the sprayed particles divert back towards the ring electrode. This is in good agreement with the simulated electric field (Fig. 1(b)) and motion angle defined as $\theta \sim 200^{\circ}$. At $300 \mathrm{~Hz}$, the stream moves in the forward direction with $\theta$ below $7^{\circ}$ and visually expands more than $150 \mathrm{~mm}$ downstream, this forwarding movement indicates that the net charge is reduced and electrostatic force has been dramatically weakened. Remarkably, the spray direction is stable and the ring electrode does not attract any visible particles during a 30-minute continuous spray. We also noted that at the frequencies above $500 \mathrm{~Hz}$ the cone collapses and the spray stops. Lower than this threshold, the spray mode does not depend on the 
frequency, and interestingly, the spray direction does not depend on the voltage. Furthermore, in the range of $100 \mathrm{~Hz}$ to $450 \mathrm{~Hz}$, the motion angle is stable $\left(7^{\circ}-10^{\circ}\right)$ and does not rely on the frequency.

In EHDA, the interval for the sufficient accumulation of charges in the vicinity of the outer surface of the Taylor cone is the charge relaxation time, $\varepsilon \varepsilon_{0} / K^{24}$. We estimate the charge relaxation time should be approximately $27 \times 10^{-6} \mathrm{~s}$, which is two orders of magnitude smaller than the switching of electric field at the minimum time interval $\sim 10^{-3} \mathrm{~s}\left((2 f)^{-1}\right)$. This charge relaxation time is also far smaller than the hydrodynamic time $L R^{2} / Q$ in which charges are confined in the outer of the Taylor cone; $L$ and $R$ are respectively the axial and radial length scales in the jet. Thus, as the switching frequency of electric field becomes comparable with the hydrodynamic time, the liquid surface may fail to follow the induced Maxwell stress to form a stable cone, and the spray quality quickly drops. With $Q=1 \mathrm{ml} / \mathrm{h}, L \sim 1 \mathrm{~mm}$ and $R \sim 17 \mu \mathrm{m}$, the upper limit for frequency is estimated as $480 \mathrm{~Hz}$ which agrees well with our experiment result.

In the optimal frequency range of $100 \mathrm{~Hz}$ to $450 \mathrm{~Hz}$ when the jet stream stably moves forward, the momentum of sprayed particles and their induced space charge are dominant factors preventing them from moving back to the ring electrode. Therefore, the recombination occurs at a specific distance from the capillary tip can be expressed by a length scale $\delta$ (Fig. 3(b)). Because of the reversed electric field, this length scale $\delta$ should be smaller than that estimated for electrohydrodynamic ac ionic winds recombined in classical nozzle-ring configuration ${ }^{25}$, i.e., $\delta<\delta_{\text {air }} \sim(\eta V / \kappa \pi f)^{1 / 2} \sim 30$ mm, where $\kappa^{2} \sim 0.1$ the dimensionless parameter for the ion recombination rates and ion screening, $\eta \sim 10^{-4} \mathrm{~m}^{2} / \mathrm{Vs}$ the ion mobility, $V=4.2 \mathrm{kV}$ and $f$ $=450 \mathrm{~Hz}$. Additionally, the frequency range from $50 \mathrm{~Hz}$ to $100 \mathrm{~Hz}$ in our work considered as the shifting regime from dc driven to ac driven spray is also reasonably smaller than reported for ac ion wind as $\sim 1500 \mathrm{~Hz}^{26}$.

Figure 4(a) shows the charge of the particle flow measured by an electrometer probe (3608 TSI) placed $200 \mathrm{~mm}$ downstream from the capillary tip for the experiment shown in Fig. 3(c). The charge is approximately $200 \mathrm{fA}$ for the ac mode at $100 \mathrm{~Hz}$. This is $97.5 \%$ reduction from the charge when the dc voltage is applied, is close to the noise level of the ambient air during the experiment and supports the aforementioned mixing mechanism of positive and negative particles. Additionally, figure 4(b) compares the measurements of the particle size distributions measured by aerosol spectrometer (TSI 3340). In the dc spray, as the majority of charged particles diverts to the ring electrode, a few particles are detected downstream whereas in the ac mode a high particle concentration sizing $100-500 \mathrm{~nm}$ are recorded, proving that charge reduced nanoparticles are efficiently generated and delivered. 

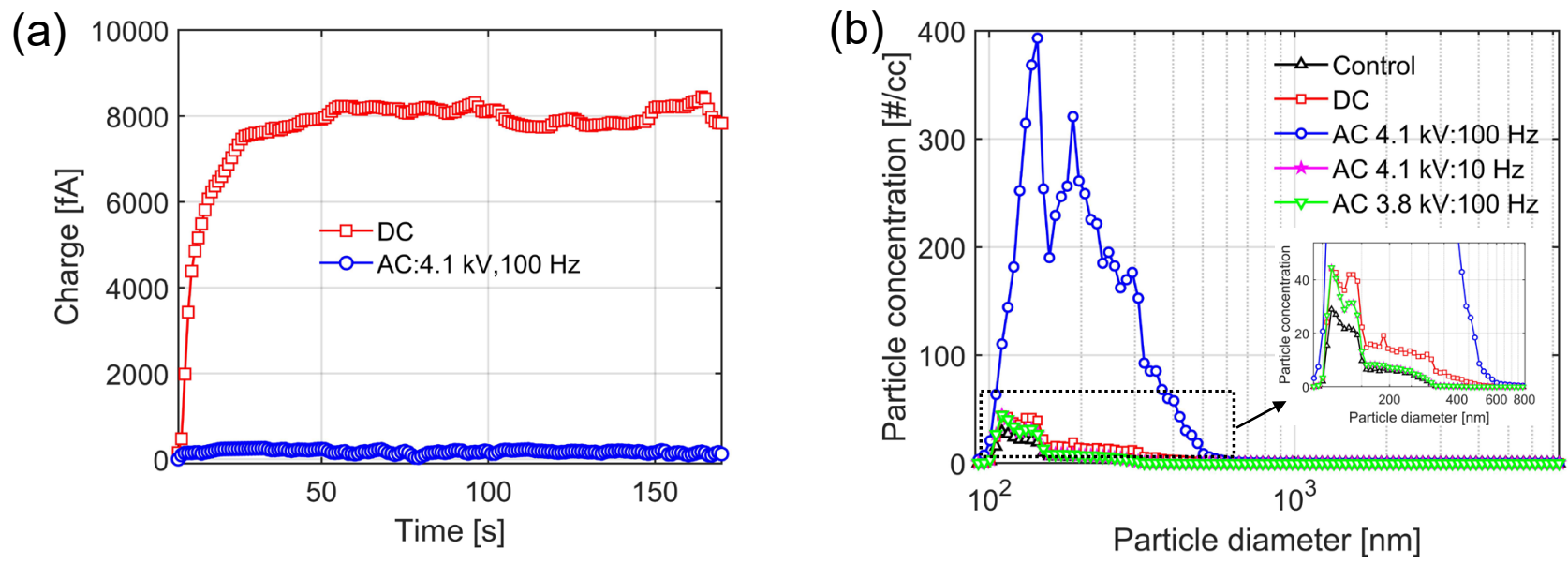

Figure 4. a) Comparision of charge measurement at downstream for dc and ac modes, b) Particle size measurement of control, dc, and ac modes, indicating the paricle size distribution is in the range of 100 to $500 \mathrm{~nm}$ (control: particle concentration measured electrospray stops).

In conclusion, we present sub-kHz electrohydrodynamic atomization simultaneously generates and delivers stable jet stream of charge reduced nanoparticles. The backward ring-nozzle configuration utilizing low frequency ac voltage in our EHDA eliminates the need for a collector electrode, which is essential in most existing EHDA systems. This unique advantage would enable the effective delivery of nanoparticles for drug delivery applications. The experimental results show the formation of the steady Taylor cone and jet stream at the optimal frequency range of $100 \sim 450 \mathrm{~Hz}$ with ac voltage range of $3.8 \sim 4.1 \mathrm{kV}$. This relatively low driving voltage and frequency enable practical developments for drug delivery and nanomedicine applications where the generation of the stable jet stream of charge reduced nanoparticles is imperative.

\section{Supplementary Material}

See the supplementary material for the measurement of particle size in different humidity conditions.

\section{Acknowledgement}

V. T. D. thanks Griffith University New Researcher Grant: Bipolar electrostatic atomizing system - fundamental study and application

\section{REFERENCES}

1. M. B. Dolovich and R. Dhand, The Lancet 377 (9770), 1032-1045 (2011).

2. J. W. Yoo, D. J. Irvine, D. E. Discher and S. Mitragotri, Nat Rev Drug Discov 10 (7), 521-535 (2011).

3. A. M. Gañán-Calvo, R. González-Prieto, P. Riesco-Chueca, M. A. Herrada and M. Flores-Mosquera, Nature Physics 3 (10), 737-742 (2007).

4. Y. Yu, L. Shang, W. Gao, Z. Zhao, H. Wang and Y. Zhao, Angew Chem Int Ed Engl 56 (40), 12127-12131 (2017).

5. J. U. Park, M. Hardy, S. J. Kang, K. Barton, K. Adair, D. K. Mukhopadhyay, C. Y. Lee, M. S. Strano, A. G. Alleyne, J.

G. Georgiadis, P. M. Ferreira and J. A. Rogers, Nat Mater 6 (10), 782-789 (2007).

6. L. Shang, Y. Cheng and Y. Zhao, Chem Rev 117 (12), 7964-8040 (2017).

7. S. Kavadiya and P. Biswas, Journal of Aerosol Science 125, 182-207 (2018).

8. N. Chetwani, C. A. Cassou, D. B. Go and H. C. Chang, J Am Soc Mass Spectrom 21 (11), 1852-1856 (2010). 
9. W. D. Ristenpart, J. C. Bird, A. Belmonte, F. Dollar and H. A. Stone, Nature 461 (7262), 377-380 (2009).

10. R. T. Collins, J. J. Jones, M. T. Harris and O. A. Basaran, Nature Physics 4 (2), 149-154 (2007).

11. P. R. Chiarot, P. Sullivan and R. Ben Mrad, Journal of Microelectromechanical Systems 20 (6), 1241-1249 (2011).

12. Q. Zhou, P. Tang, S. S. Y. Leung, J. G. Y. Chan and H. K. Chan, Adv Drug Deliver Rev 75, 3-17 (2014).

13. L. Xu and D. Sun, Applied Physics Letters 102 (2) (2013).

14. N. Rangaraj, S. R. Pailla and S. Sampathi, Pulm Pharmacol Ther 54, 1-21 (2019).

15. J. Fernandez de la Mora and C. Barrios-Collado, Aerosol Science and Technology 51 (6), 778-786 (2017).

16. J. P. Borra, D. Camelot, K.-L. Chou, P. J. Kooyman, J. C. M. Marijnissen and B. Scarlett, Journal of Aerosol Science 30

(7), 945-958 (1999).

17. J. P. Borra, D. Camelot, J. C. M. Marijnissen and B. Scarlett, Journal of Electrostatics 40-41, 633-638 (1997).

18. V. T. Dau and T. Terebessy, US Patent Patent No. US9937508 (feb 12 2014).

19. L. Y. Yeo, D. Lastochkin, S. C. Wang and H. C. Chang, Physical Review Letters 92, 133902-133901 (2004).

20. P. Wang, S. Maheshwari and H. C. Chang, Phys Rev Lett 96 (25), 254502 (2006).

21. D. B. Bober and C.-H. Chen, Journal of Fluid Mechanics 689, 552-563 (2011).

22. A. Jaworek, A. T. Sobczyk and A. Krupa, Journal of Aerosol Science 125, 57-92 (2018).

23. M. G.M.H., P. H. W. Vercoulen, J. C. M. Marijnissen and B. Scarlett, Journal of Aerosol Science 23 (1), $37-49$ (1992).

24. S. Maheshwari and H.-C. Chang, Applied Physics Letters 89 (23) (2006).

25. A. M. Drews, L. Cademartiri, G. M. Whitesides and K. J. M. Bishop, Journal of Applied Physics 114 (2013).

26. V. T. Dau, T. X. Dinh, C.-D. Tran, T. Terebessy and T. T. Bui, Experimental Thermal and Fluid Science 97 (2018). 